\title{
On the Lie transformation algebra of monoids in symmetric monoidal categories
}

\author{
ABhishek BanerJee
}

ABstRact - We define the Lie transformation algebra of a (not necessarily associative) monoid object $A$ in a $K$-linear symmetric monoidal category $(\mathbf{C}, \otimes, \mathbf{1})$, where $K$ is a field. When $A$ is associative and satisfies certain conditions, we describe explicity the Lie transformation algebra and inner derivations of $A$. Additionally, we also show that derivations preserve the nucleus of the monoid $A$

Mathematics SubJect Classification (2010). 17A36, $18 \mathrm{D} 10$.

KEYWORDS. Inner derivations, Lie transformation algebra.

\section{Introduction}

Given an associative Z-algebra $A$ and an element $a \in A$, the morphism $D_{a}: A \longrightarrow A$ defined by $D_{a}(x):=a x-x a$ defines an inner derivation on $A$. However, if $A$ is not associative, the morphism $D_{a}$ is not necessarily a derivation. For nonassociative algebras, a theory of inner derivations has been developed by Schafer [4]. The purpose of this paper is to extend this theory to monoids over a $K$-linear symmetric monoidal category $(\mathbf{C}, \otimes, \mathbf{1})$, where $K$ is a field.

More precisely, let $A$ be a (not necessarily associative) unital monoid object in a $K$-linear symmetric monoidal category $(\mathbf{C}, \otimes, \mathbf{1})$. A morphism $f: 1 \longrightarrow A$ induces a morphism $L_{A}(f): A \longrightarrow A$ (resp. $R_{A}(f): A \longrightarrow A$ ) by left multiplication (resp. right multiplication) on the monoid $A$ (in the sense of (2.9)). We consider the subspace $\mathcal{L}(A)(\operatorname{resp} . \mathcal{R}(A))$ of $\operatorname{Hom}(A, A)$ generated by morphisms of the form $L_{A}(f)\left(\operatorname{resp} . R_{A}(f)\right)$. Then, we start by defining the Lie transformation algebra $\mathscr{L}(A)$ of $A$ to be the smallest Lie algebra con-

(*) Indirizzo dell'A.: Collège de France, 3, rue d'Ulm, 75231, Paris cedex 05, France.

E-mail: abhishekbanerjee1313@gmail.com 
taining the subspace $\mathcal{L}(A)+\mathcal{R}(A)$ of $\operatorname{Hom}(A, A)$. If $D$ is a derivation on $A$ (see Definition 2.10), we will say that $D$ is an inner derivation if $D \in \mathscr{L}(A)$.

When $A$ is an associative monoid object, we show that the Lie transformation algebra $\mathscr{L}(A)$ of $A$ is actually equal to $\mathcal{L}(A)+\mathcal{R}(A)$. In particular, if $A$ is associative and has no left (or right) "absolute divisors of zero", we show that a derivation $D$ on $A$ is inner if and only if $D$ is of the form $D=L_{A}(f)-R_{A}(f)$ for some morphism $f: 1 \longrightarrow A$. Moreover, we verify that for any (not necessarily associative) monoid $A$, the collection of inner derivations is always an ideal in the Lie algebra $\operatorname{Der}(A)$ of derivations on $A$. Finally, we also show that if $f: 1 \longrightarrow A$ is a morphism in the nucleus of $A$ (see (2.13)), for any derivation $D \in \operatorname{Der}(A), D \circ f$ is also in the nucleus of $A$.

We mention here that the notion of derivations on monoid objects appears elsewhere in the literature (see, for instance, Baues, Jibladze and Tonks [1]). For more on derivations and nonassociative algebras, we refer the reader to Jacobson [2] and Schafer [4], [5].

\section{Derivations on monoids}

Let $(\mathbf{C}, \otimes, \mathbf{1})$ be a $K$-linear symmetric monoidal category. Since $\mathbf{C}$ is symmetric, for every pair $X, Y$ of objects in $\mathbf{C}$, we have an isomorphism: $t_{X, Y}: X \otimes Y \stackrel{\cong}{\longrightarrow} Y \otimes X$ such that $t_{X, Y} \circ t_{Y, X}=1_{Y \otimes X}$ and $t_{Y, X} \circ t_{X, Y}=1_{X \otimes Y}$. When there is no danger of confusion, we shall omit the subscripts and simply write $t: X \otimes Y \stackrel{\cong}{\longrightarrow} Y \otimes X$. Further, for any object $X$ in $\mathbf{C}$, we have two isomorphisms $l_{X}: X \stackrel{\cong}{\rightrightarrows} 1 \otimes X$ and $r_{X}: X \stackrel{\cong}{\longrightarrow} X \otimes 1$ satisfying $r_{X}=t l_{X}$.

Given $(\mathbf{C}, \otimes, \mathbf{1})$, we shall let $\operatorname{Mon}(\mathbf{C})$ denote the category of unital, not necessarily associative, monoids object in $\mathbf{C}$. For any monoid $A$ in $\mathbf{C}$, we will denote by $m_{A}: A \otimes A \longrightarrow A$ and $e_{A}: 1 \longrightarrow A$ resp. the "multiplication map" and the "unit map" on the monoid $A$. We start by defining the notion of a derivation on $A$.

Definition 2.1. Let $A$ be an object of $M o n(\mathbf{C})$. A morphism $D: A \longrightarrow A$ is referred to as a derivation on $A$ if it satisfies the following condition:

$$
m_{A} \circ(D \otimes 1+1 \otimes D)=D \circ m_{A}: A \otimes A \longrightarrow A
$$

Given derivations $D, D^{\prime}$ of a monoid $A$, it may be easily verified that the commutator $\left[D, D^{\prime}\right]:=D \circ D^{\prime}-D^{\prime} \circ D$ is also a derivation on $A$. Then, since the category $\mathbf{C}$ is $K$-linear, the space $\operatorname{Der}(A)$ of derivations on $A$ is a Lie algebra. 
Further, for any monoid $A$, given a morphism $f: 1 \longrightarrow A$, we define:

$$
\begin{aligned}
L_{A}(f) & :=\left(A \underset{l_{A}}{\stackrel{l}{\longrightarrow}} 1 \otimes A \stackrel{f \otimes 1}{\longrightarrow} A \otimes A \stackrel{m_{A}}{\longrightarrow} A\right) \\
R_{A}(f) & :=\left(A \stackrel{r_{A}}{\cong} A \otimes 1 \stackrel{1 \otimes f}{\longrightarrow} A \otimes A \stackrel{m_{A}}{\longrightarrow} A\right) \\
& :=\left(A \stackrel{l_{A}}{\cong} 1 \otimes A \stackrel{f \otimes 1}{\longrightarrow} A \otimes A \stackrel{m_{A} t}{\longrightarrow} A\right)
\end{aligned}
$$

We denote by $\mathcal{L}(A)($ resp. $\mathcal{R}(A))$ the subspace of $\operatorname{Hom}(A, A)$ generated by morphisms of the form $L_{A}(f)$ (resp. $R_{A}(f)$ ) where $f \in \operatorname{Hom}(1, A)$. We will say that $f: 1 \longrightarrow A$ is a left (resp. right) absolute divisor of zero if $L_{A}(f)=0\left(\operatorname{resp} . R_{A}(f)=0\right)$.

Suppose that $\mathscr{C} 6:=\mathcal{L}(A)+\mathcal{R}(A) \subseteq \operatorname{Hom}(A, A)$. If we define the sequence of spaces $\left\{\mathscr{C}_{i}\right\}_{i \in \mathrm{N}}$ as follows:

$$
\mathscr{C}_{1}=\mathscr{C l} \quad \mathscr{C}_{i}:=\left[\mathscr{C}_{1}, \mathscr{C l}_{i-1}\right], \quad i=2,3, \ldots .
$$

then, as in $[4, \S 2]$, the space $\mathscr{B}(A):=\mathscr{M}_{1}+\mathscr{M}_{2}+\ldots$ is the smallest Lie algebra containing $\mathscr{b}_{1}=\mathscr{l}$. Then $\mathscr{L}(A)$ is referred to as the Lie transformation algebra of $A$. Following [4], we will say that a derivation $D: A \longrightarrow A$ is inner if $D \in \mathscr{B}(A)$.

In particular, suppose that $A$ is an associative monoid. Then, for any morphism $f: 1 \longrightarrow A$, it is known (see $[1, \S 4]$ ) that $L_{A}(f)-R_{A}(f) \in$ $\operatorname{Hom}(A, A)$ is a derivation on $A$. Further, for any $f, g: 1 \longrightarrow A$, it follows from associativity of $A$ that either of the compositions $L_{A}(f) \circ R_{A}(g)$ and $R_{A}(g) \circ L_{A}(f)$ is equal to the composition

$$
\begin{aligned}
& L_{A}(f) R_{A}(g)= \\
& R_{A}(g) L_{A}(f): A \stackrel{\cong}{\longrightarrow} 1 \otimes(A \otimes 1) \stackrel{f \otimes(1 \otimes g)}{\longrightarrow} A \otimes(A \otimes A) \stackrel{m_{A^{\circ}\left(1 \otimes m_{A}\right)}^{\longrightarrow} A}{\longrightarrow}
\end{aligned}
$$

i.e., we have $\left[L_{A}(f), R_{A}(g)\right]=0$. We will denote by $m(f, g)$ the morphism $m(f, g): 1 \stackrel{\cong}{\longrightarrow} 1 \otimes 1 \stackrel{f \otimes g}{\longrightarrow} A \otimes A \stackrel{m_{A}}{\longrightarrow} A$. We now have the following result.

Proposition 2.2. Let $A$ be an associative monoid object in $(\mathbf{C}, \otimes, \mathbf{1})$. Then, the Lie transformation algebra $\mathscr{B}(A)$ of $A$ is given by

$$
\mathscr{L}(A)=\mathcal{L}(A)+\mathcal{R}(A)
$$

Further, if $A$ has no left (resp. right) absolute divisor of zero, then $D \in \operatorname{Hom}(A, A)$ is an inner derivation if and only if $D=L_{A}(f)-R_{A}(f)$ for some $f: 1 \longrightarrow A$.

Proof. Let us choose elements $L_{A}(f)+R_{A}(g), L_{A}\left(f^{\prime}\right)+R_{A}\left(g^{\prime}\right) \in \mathcal{L}(A)+$ $\mathcal{R}(A)$ for morphisms $f, f^{\prime}, g, g^{\prime}: 1 \longrightarrow A$. Then, since $\left[L_{A}(f), R_{A}\left(g^{\prime}\right)\right]=$ 
$\left[L_{A}\left(f^{\prime}\right), R_{A}(g)\right]=0$, it follows that

$$
\left[L_{A}(f)+R_{A}(g), L_{A}\left(f^{\prime}\right)+R_{A}\left(g^{\prime}\right)\right]=\left[L_{A}(f), L_{A}\left(f^{\prime}\right)\right]+\left[R_{A}(g), R_{A}\left(g^{\prime}\right)\right]
$$

We now notice that:

$$
\begin{aligned}
& L_{A}(f) L_{A}\left(f^{\prime}\right) \\
& =\left(A \stackrel{\cong}{\longrightarrow} 1 \otimes A \stackrel{f^{\prime} \otimes 1}{\longrightarrow} A \otimes A \stackrel{m_{A}}{\longrightarrow} A \stackrel{\cong}{\longrightarrow} 1 \otimes A \stackrel{f \otimes 1}{\longrightarrow} A \otimes A \stackrel{m_{A}}{\longrightarrow} A\right) \\
& \text { (2.7) }=\left(A \stackrel{\cong}{\longrightarrow} 1 \otimes(1 \otimes A) \stackrel{f \otimes\left(f^{\prime} \otimes 1\right)}{\longrightarrow} A \otimes(A \otimes A) \stackrel{m_{A^{\circ}\left(1 \otimes m_{a}\right)}^{\longrightarrow}}{\longrightarrow} A\right) \\
& =\left(A \stackrel{\cong}{\longrightarrow}(1 \otimes 1) \otimes A \stackrel{\left(f \otimes f^{\prime}\right) \otimes 1}{\longrightarrow}(A \otimes A) \otimes A \stackrel{m_{A} \circ\left(m_{a} \otimes 1\right)}{\longrightarrow} A\right)=L_{A}\left(m\left(f, f^{\prime}\right)\right)
\end{aligned}
$$

Hence, it follows that $\left[L_{A}(f), L_{A}\left(f^{\prime}\right)\right]=L_{A}\left(m\left(f, f^{\prime}\right)-m\left(f^{\prime}, f\right)\right)$. Similarly, we may show that $R_{A}(g) R_{A}\left(g^{\prime}\right)=R_{A}\left(m\left(g^{\prime}, g\right)\right)$ and hence $\left[R_{A}(g), R_{A}\left(g^{\prime}\right)\right]=$ $R_{A}\left(m\left(g^{\prime}, g\right)-m\left(g, g^{\prime}\right)\right)$. From (2.6), it follows that

$$
\begin{aligned}
& {\left[L_{A}(f)+R_{A}(g), L_{A}\left(f^{\prime}\right)+R_{A}\left(g^{\prime}\right)\right]=} \\
& \quad L_{A}\left(m\left(f, f^{\prime}\right)-m\left(f^{\prime}, f\right)\right)-R_{A}\left(m\left(g^{\prime}, g\right)-m\left(g, g^{\prime}\right)\right)
\end{aligned}
$$

and hence $\mathcal{L}(A)+\mathcal{R}(A)$ is a Lie algebra. Since $\mathscr{L}(A)$ is the smallest Lie algebra containing $\mathcal{L}(A)+\mathcal{R}(A)$, we have $\mathscr{L}(A)=\mathcal{L}(A)+\mathcal{R}(A)$.

We now suppose that $L_{A}(f)+R_{A}(g) \in \mathscr{B}(A)$ is a derivation. Since $L_{A}(g)-R_{A}(g)$ is a derivation as mentioned before, so is $L_{A}(f+g)=$ $\left(L_{A}(f)+R_{A}(g)\right)+\left(L_{A}(g)-R_{A}(g)\right)$. Since $A$ is associative, the following commutative diagram
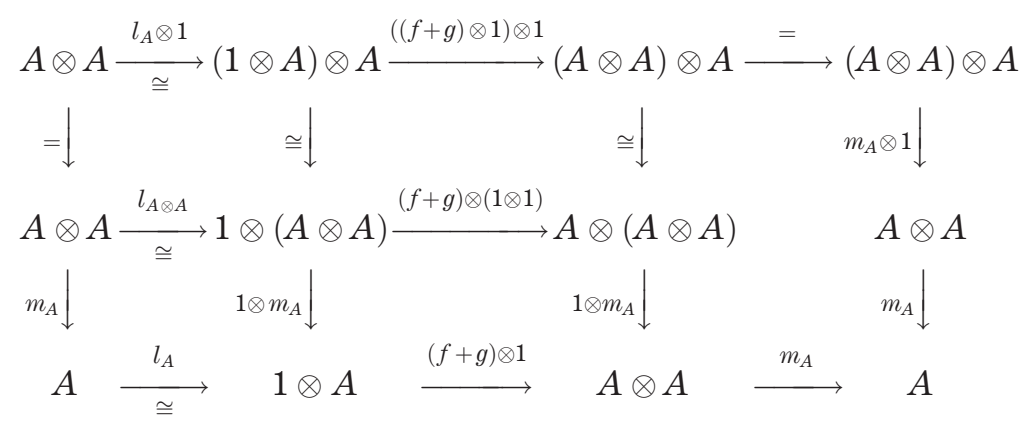

shows that $m_{A} \circ\left(L_{A}(f+g) \otimes 1\right)=L_{A}(f+g) \circ m_{A}: A \otimes A \longrightarrow A$. Since $L_{A}(f+g)$ is a derivation, it follows from $(2.1)$ that $m_{A} \circ\left(1 \otimes L_{A}(f+g)\right)=0$. On the other hand, we note that

$$
\begin{array}{r}
0=m_{A} \circ\left(1 \otimes L_{A}(f+g)\right) \circ\left(e_{A} \otimes 1\right) \circ l_{A}=L_{A}\left(m\left(e_{A}, f+g\right)\right)= \\
L_{A}\left(e_{A}\right) L_{A}(f+g)=L_{A}(f+g)
\end{array}
$$


and hence $L_{A}(f+g)=0$. Now, if $A$ has no left absolute divisors of zero, it follows that $L_{A}(f+g)=0$ and the inner derivation $L_{A}(f)+R_{A}(g) \in \mathscr{B}(A)$ is actually of the form $L_{A}(f)+R_{A}(g)=R_{A}(g)-L_{A}(g)=L_{A}(-g)-$ $R_{A}(-g)$. The result follows similarly for the case of no right absolute divisors of zero.

Proposition 2.3. Let $A$ be an object of $\operatorname{Mon}(\mathbf{C})$. Then, the space $\mathscr{B}(A) \cap \operatorname{Der}(A)$ of inner derivations on $A$ is an ideal in the Lie algebra $\operatorname{Der}(A)$.

Proof. We choose a morphism $f: 1 \longrightarrow A$ and some $D \in \operatorname{Der}(A)$. Our first objective is to show that $\left[D, L_{A}(f)\right]=D \circ L_{A}(f)-L_{A}(f) \circ D=$ $L_{A}(D \circ f)$. For this, we note that:

$$
\begin{aligned}
D \circ L_{A}(f) & =D \circ m_{A} \circ(f \otimes 1) \circ l_{A} \\
& =m_{A} \circ(D \otimes 1+1 \otimes D) \circ(f \otimes 1) \circ l_{A} \\
& =m_{A} \circ((D \circ f) \otimes 1) \circ l_{A}+m_{A} \circ(1 \otimes D) \circ(f \otimes 1) \circ l_{A} \\
& =L_{A}(D \circ f)+m_{A} \circ(f \otimes 1) \circ(1 \otimes D) \circ l_{A} \\
& =L_{A}(D \circ f)+m_{A} \circ(f \otimes 1) \circ l_{A} \circ D \\
& =L_{A}(D \circ f)+L_{A}(f) \circ D
\end{aligned}
$$

It follows from (2.11) that $[D, \mathcal{L}(A)] \subseteq \mathcal{L}(A)$. Similarly, we may show that $[D, \mathcal{R}(A)] \subseteq \mathcal{R}(A)$. It follows, therefore, that for $\mathscr{C l}_{1}=\mathscr{C l}=\mathcal{L}(A)+\mathcal{R}(A)$, $\left[D, \mathscr{L}_{1}\right] \subseteq \mathscr{M}_{1}$.

We now suppose that $\left[D, \mathscr{L}_{j}\right] \subseteq \mathscr{L}_{j}$ for all $j \leq i$ for some given $i$. Then, given any element $D^{\prime} \in \mathscr{L}_{i+1}$, by definition of $\mathscr{L}_{i+1}$ in (2.3), $D^{\prime}$ may be written as a sum $D^{\prime}=\sum_{l=1}^{k} D_{l}^{\prime}$ with each $D_{l}^{\prime} \in\left[\mathscr{L}_{1}, \mathscr{L}_{i}\right]$. We now note that for each $1 \leq l \leq k$, we have

$$
\begin{aligned}
& {\left[D, D_{l}^{\prime}\right] \in\left[D,\left[\mathscr{H}_{1}, \mathscr{C}_{i}\right]\right] \subseteq\left[\mathscr{H}_{1},\left[D, \mathscr{L}_{i}\right]\right]+\left[\mathscr{C}_{i},\left[D, \mathscr{C O}_{1}\right]\right] \subseteq} \\
& {\left[\mathscr{M b}_{1}, \mathscr{L}_{i}\right]+\left[\mathscr{M b}_{i}, \mathscr{C b}_{1}\right]=\mathscr{M b}_{i+1}}
\end{aligned}
$$

From (2.12), it follows that $\left[D, \mathscr{C}_{i+1}\right] \subseteq \mathscr{C}_{i+1}$ and hence $\left[D, \mathscr{C}_{i}\right] \subseteq \mathscr{L}_{i}$ for all $i \geq 1$ by induction. It follows that $[D, \mathscr{L}(A)] \subseteq \mathscr{L}(A)$. Since $[D, \operatorname{Der}(A)] \subseteq \operatorname{Der}(A)$, it follows that $[D, \mathscr{L}(A) \cap \operatorname{Der}(A)] \subseteq \mathscr{L}(A) \cap \operatorname{Der}(A)$.

REMARK 2.4. It follows from Proposition 2.3 that if $A$ is a monoid such that the derivation algebra $\operatorname{Der}(A)$ is simple (as a Lie algebra) and there exist non zero inner derivations of $A$, then every derivation of $A$ is inner. 
Given a monoid object $A$, we will say that a morphism $f: 1 \longrightarrow A$ is in the nucleus of $A$ if each of the following three morphisms is identically 0 :

$$
\begin{aligned}
& A_{0}(f):=\left(A \otimes A \stackrel{l_{A} \otimes 1}{\cong}(1 \otimes A) \otimes A \stackrel{(f \otimes 1) \otimes 1}{\longrightarrow}(A \otimes A) \otimes A \stackrel{m_{A}\left(m_{A} \otimes 1-\left(1 \otimes m_{A}\right) a\right)}{\longrightarrow} A\right) \\
& A_{1}(f):=\left(A \otimes A \stackrel{1 \otimes l_{A}}{\cong} A \otimes(1 \otimes A) \stackrel{1 \otimes(f \otimes 1)}{\longrightarrow} A \otimes(A \otimes A) \stackrel{m_{A}\left(\left(m_{A} \otimes 1\right) a^{-1}-1 \otimes m_{A}\right)}{\longrightarrow} A\right) \\
& A_{2}(f):=\left(A \otimes A \stackrel{1 \otimes r_{A}}{\cong} A \otimes(A \otimes 1) \stackrel{1 \otimes(1 \otimes f)}{\longrightarrow} A \otimes(A \otimes A) \stackrel{m_{A}\left(\left(m_{A} \otimes 1\right) a^{-1}-1 \otimes m_{A}\right)}{\longrightarrow} A\right)
\end{aligned}
$$

where $a$ is the natural isomorphism $a:(A \otimes A) \otimes A \stackrel{\cong}{\longrightarrow} A \otimes(A \otimes A)$. The set of all morphisms in the nucleus of $A$ will be denoted by $N u c(A)$ (compare $[3, \S 1.13])$. Clearly, if $A$ is associative, $N u c(A)=\operatorname{Hom}(1, A)$.

Proposition 2.5. Let $A$ be an object of $\operatorname{Mon}(\mathbf{C})$. Let $D$ be a derivation on $A$ and let $f: 1 \longrightarrow A$ be an element of the nucleus of $A$. Then, $D \circ f \in N u c(A)$.

Proof. Since $f \in N u c(A)$, we know that $A_{0}(f)=A_{1}(f)=A_{2}(f)=0$ as defined in (2.13). We proceed as follows:

$$
\begin{aligned}
& A_{0}(D \circ f) \\
& =m_{A}\left(m_{A} \otimes 1-\left(1 \otimes m_{A}\right) a\right)((D \circ f \otimes 1) \otimes 1)\left(l_{A} \otimes 1\right) \\
& =m_{A}\left(m_{A} \otimes 1-\left(1 \otimes m_{A}\right) a\right)((D \otimes 1) \otimes 1)((f \otimes 1) \otimes 1)\left(l_{A} \otimes 1\right) \\
& =m_{A}\left(\left(D m_{A}-m_{A}(1 \otimes D)\right) \otimes 1\right)((f \otimes 1) \otimes 1)\left(l_{A} \otimes 1\right) \\
& -m_{A}(D \otimes 1)\left(1 \otimes m_{A}\right) a((f \otimes 1) \otimes 1)\left(l_{A} \otimes 1\right) \\
& =\left(m_{A}(D \otimes 1)\left(m_{A} \otimes 1\right)-m_{A}\left(m_{A} \otimes 1\right)((1 \otimes D) \otimes 1)\right. \\
& \left.-D m_{A}\left(1 \otimes m_{A}\right) a+m_{A}\left(1 \otimes D m_{A}\right) a\right) \circ((f \otimes 1) \otimes 1)\left(l_{A} \otimes 1\right) \\
& =\left(D m_{A}\left(m_{A} \otimes 1\right)-m_{A}(1 \otimes D)\left(m_{A} \otimes 1\right)-m_{A}\left(m_{A} \otimes 1\right)((1 \otimes D) \otimes 1)\right. \\
& \left.-D m_{A}\left(1 \otimes m_{A}\right) a+m_{A}\left(1 \otimes D m_{A}\right) a\right)((f \otimes 1) \otimes 1)\left(l_{A} \otimes 1\right) \\
& =D \circ A_{0}(f)-m_{A}\left(\left(m_{A} \otimes 1\right)((1 \otimes 1) \otimes D)+\left(m_{A} \otimes 1\right)((1 \otimes D) \otimes 1)\right. \\
& \left.-\left(1 \otimes D m_{A}\right) a\right)((f \otimes 1) \otimes 1)\left(l_{A} \otimes 1\right) \\
& =-m_{A}\left(m_{A} \otimes 1\right)((1 \otimes D) \otimes 1+(1 \otimes 1) \otimes D)((f \otimes 1) \otimes 1)\left(l_{A} \otimes 1\right) \\
& \quad+m_{A}\left(1 \otimes m_{A}\right)(1 \otimes(D \otimes 1)+1 \otimes(1 \otimes D)) a((f \otimes 1) \otimes 1)\left(l_{A} \otimes 1\right) \\
& =m_{A}\left(\left(1 \otimes m_{A}\right) a-m_{A} \otimes 1\right)((f \otimes 1) \otimes 1)((1 \otimes D) \otimes 1)\left(l_{A} \otimes 1\right) \\
& \quad+m_{A}\left(\left(1 \otimes m_{A}\right) a-m_{A} \otimes 1\right)((f \otimes 1) \otimes 1)((1 \otimes 1) \otimes D)\left(l_{A} \otimes 1\right) \\
& =-A_{0}(f) \circ(D \otimes 1)-A_{0}(f) \circ(1 \otimes D)=0
\end{aligned}
$$


Similarly, one may check that both $A_{1}(D \circ f)$ and $A_{2}(D \circ f)$ are 0 . Hence, $D \circ f \in N u c(A)$.

\section{REFERENCES}

[1] H.-J. Baues, M. Jibladze, A. Tonks, Cohomology of monoids in monoidal categories. Operads: Proceedings of Renaissance Conferences (Hartford, CT/ Luminy, 1995), 137-165, Contemp. Math., 202, Amer. Math. Soc., Providence, RI, 1997.

[2] N. JaCOBSON, Derivation algebras and multiplication algebras of semi-simple Jordan algebras. Ann. of Math. (2) 50 (1949), 866-874.

[3] O. Loos, P. H. Petersson, M. L. Racine, Inner derivations of alternative algebras over commutative rings. Algebra Number Theory 2 (2008), no. 8, 927-968.

[4] R. D. SCHAFER, Inner derivations of non-associative algebras. Bull. Amer. Math. Soc. 55 (1949), 769-776.

[5] R. D. Schafer, An introduction to nonassociative algebras. Pure and Applied Mathematics, 22, Academic Press, New York-London 1966.

Manoscritto pervenuto in redazione il 7 Ottobre 2012. 
